\title{
ANOMALOUSLY WEAK DYNAMICAL FRICTION IN HALOS
}

\author{
J. A. Sellwood \\ Department of Physics \& Astronomy, Rutgers University \\ 136 Frelinghuysen Road, Piscataway NJ 08854-8019, USA \\ sellwood@physics.rutgers.edu \\ Victor P. Debattista \\ Astronomy Department, University of Washington \\ Box 351580, Seattle, WA 98195-1580, USA \\ debattis@astro.washington.edu
}

\begin{abstract}
A bar rotating in a pressure-supported halo generally loses angular momentum and slows down due to dynamical friction. Valenzuela \& Klypin report a counter-example of a bar that rotates in a dense halo with little friction for several Gyr, and argue that their result invalidates the claim by Debattista \& Sellwood that fast bars in real galaxies require a low halo density. We show that it is possible for friction to cease for a while should the pattern speed of the bar fluctuate upward. The reduced friction is due to an anomalous gradient in the phase-space density of particles at the principal resonance created by the earlier evolution. The result obtained by Valenzuela \& Klypin is probably an artifact of their adaptive mesh refinement method, but anyway could not persist in a real galaxy. The conclusion by Debattista \& Sellwood still stands.
\end{abstract}

Keywords: Galaxies: kinematics and dynamics — galaxies: halos — dark matter

\section{Introduction}

It is now well established that a bar rotating in a halo loses angular momentum through dynamical friction. This topic has received a lot of attention recently for two important reasons: (1) it offers a constraint on the density of the DM halo (Debattista \& Sellwood 1998, 2000), and (2) it may flatten the density cusp (Weinberg \& Katz 2002).

Both these claims have been challenged. Realistic bars in cuspy halos produce a mild density decrease at most (Holley-Bockelmann et al. 2003) or even a slight increase (Sellwood 2003), but we leave this issue 


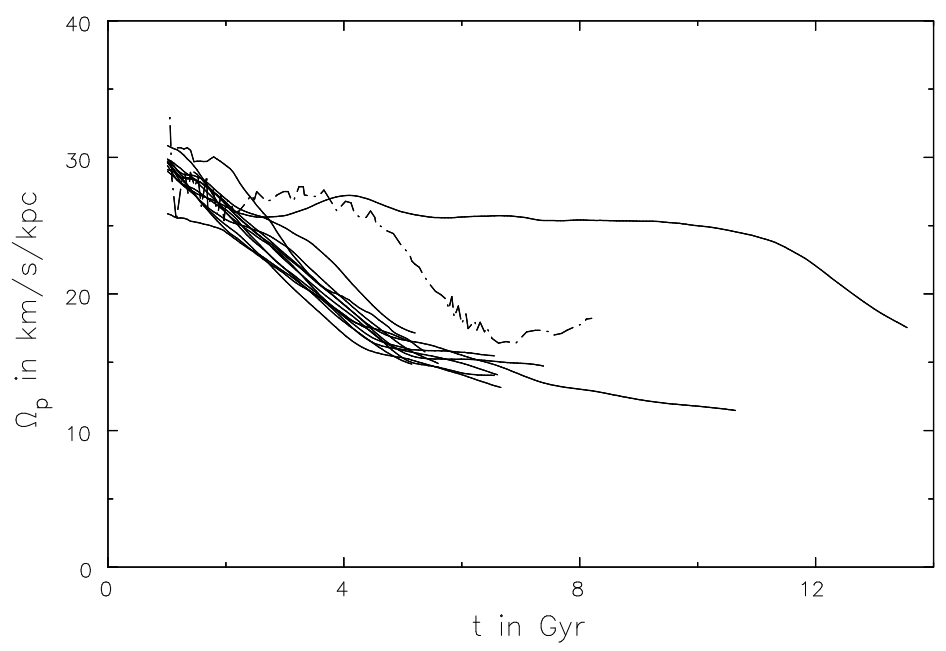

Figure 1. The time evolution of the bar pattern speed in a number of resimulations of model $\mathrm{A}_{1}$ of VK03. The evolution reported by VK03 is reproduced as the dotdashed line; all other lines are from simulations from the same initial particle load, but run with our code using many different sets of numerical parameters.

aside here and concentrate instead on the density constraint. HolleyBockelmann \& Weinberg (2005) announce a preliminary report of simulations with weak friction in halos having uniform density cores, but we focus here on the older counter-example claimed by Valenzuela \& Klypin (2003; hereafter VK03) of a bar that experiences little friction in a cusped dense halo.

VK03 kindly made available the initial positions and velocities of all the particles of their model $A_{1}$, in which the bar did not slow for 2-3 Gyrs after it had formed and settled. We have used our code (Sellwood 2003) to rerun this simulation many times, and the pattern speed evolution in many of these runs is shown in Figure 1. It is striking that in most cases, the bar slowed earlier than VK03 found, but in one anomalous case, the bar stayed fast for about 10 Gyr! The anomalous result is not a consequence of some inadequate numerical parameter, since many of the other cases are from models with parameters that bracket those of the anomalous case - i.e. longer and shorter time steps, coarser and finer grids, etc.

Note that apart from the crucial delay in the onset of friction in the case by VK03 and the one anomalous case we find, the evolution is generally very similar. In particular, whenever the bar slows, it slows 
at a similar rate. The following sections account for the discrepancies between the results shown in Fig. 1.

\section{Frictional Torque}

In a classic paper, Tremaine \& Weinberg (1984) laid out the mathematical apparatus for friction in a spherical system. Following the precepts of Lynden-Bell \& Kalnajs (1972), they derived a formula for the torque experienced by a rotating perturbation potential, $\Phi_{p}$. They work in action-angle variables (see Binney \& Tremaine 1987, §3.5). In a spherical potential, there are two non-zero actions: the total angular momentum per unit mass $L \equiv J_{\phi}$ and the radial action $J_{r}$, each associated with two separate frequencies, $\Omega$ and $\kappa$, which are generalizations to orbits of arbitrary eccentricity of the usual frequencies of Lindblad epicycles familiar from disk dynamics. In the limit that a constant amplitude perturbation rotates steadily at $\Omega_{p}$, they showed that the net LBK torque is

$$
\tau_{\mathrm{LBK}} \propto \sum_{m, k, n}\left(m \frac{\partial f}{\partial L}+k \frac{\partial f}{\partial J_{r}}\right)\left|\Phi_{m n k}\right|^{2} \delta\left(n \Omega+k \kappa-m \Omega_{p}\right),
$$

where $f$ is the usual distribution function and $\Phi_{m n k}$ is a Fourier coefficient of the perturbing potential. The Dirac delta function implies that the net torque is the sum of the separate contributions from resonances, where $n \Omega+k \kappa=m \Omega_{p}$. Because the bar pattern speed decreases, as a result of the frictional torque, this expression needs to be generalized to a time-dependent forcing (see Weinberg 2004), but the revised expression for the torque still contains the same derivatives of the distribution function.

Lynden-Bell (1979) offered a clear insight into how an orbit is affected when close to a resonance. The unperturbed orbit, which is a rosette in an inertial reference frame, closes in any frame that rotates at the rate

$$
\Omega^{\prime}=\Omega+k \kappa / m,
$$

for any pair $k, m$. [See e.g. Kalnajs (1977) for illustrations of several of the most important shapes.] When the pattern speed of the bar is close to $\Omega^{\prime}$ for some pair $k, m$, the orbit can be regarded as a closed figure that precesses at the slow rate

$$
\Omega_{s} \equiv\left(\Omega^{\prime}-\Omega_{p}\right) \ll \Omega_{p}
$$

Under these circumstances, the "fast action" is adiabatically invariant, while the "slow action" can suffer a large change. Things are particularly 

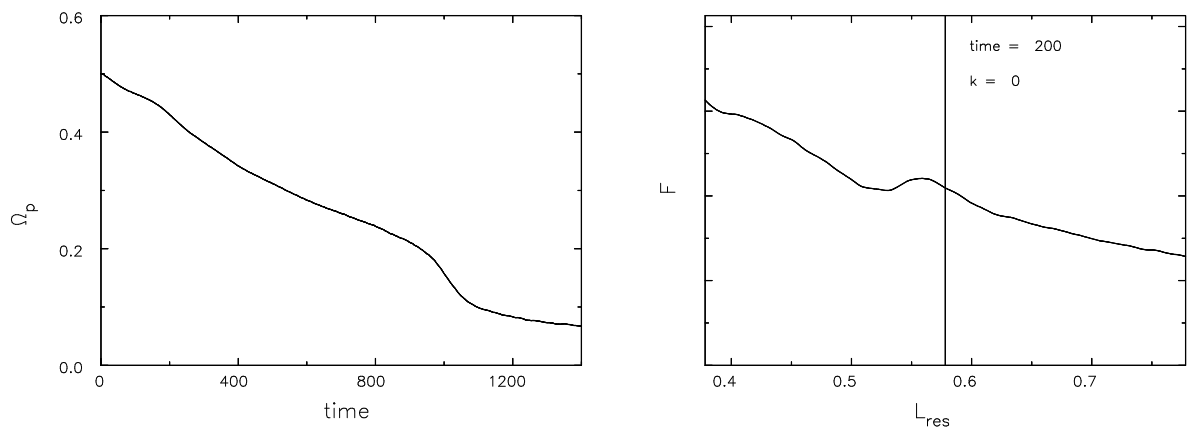

Figure 2. Left: The time evolution of the bar pattern speed in the restricted simulation discussed in $\S 3$. This simulation employs $10 \mathrm{M}$ particles. Right: The mean density of particles as a function of $L_{\mathrm{res}}$ at $t=200$ in the same simulation.

straightforward at corotation, where the fast action is the radial action, while the slow action that can suffer a large change is simply the angular momentum.

\section{Restricted Simulations}

Fully self-consistent simulations are complicated by evolution of the total potential, changes to the bar mass profile, etc. It is therefore easier first to try to understand "restricted" simulations in which a rigid bar rotates in halo of non-interacting test particles (Lin \& Tremaine 1983; Sellwood 2004). The particles move in a rigid halo potential that is perturbed by that of the rotating bar, and the bar is accelerated in response to the vector-sum of the non-axisymmetric forces felt by the particles. Figure 2 shows an example of the pattern speed evolution of a homogeneous ellipsoid with principal axes $1: 0.5: 0.1$ in a Hernquist (1990) halo. The bar mass is $1 \%$ of the halo mass, $M_{h}$, has a semi-major axis equal to the halo break radius $r_{h}$, and rotates initially so that it just fills its corotation circle. We use units such that $G=M_{h}=r_{h}=1$.

At intervals during the simulation, we compute $\Omega^{\prime}=\Omega+k \kappa / m$ for every particle, and calculate $F$, the average density of particles as a function $\Omega^{\prime}$. It is somewhat easier to understand a plot of $F$ as a function of the angular momentum, $L_{\text {res }}$, of a circular orbit that has the given $\Omega^{\prime}(k, m)$.

The right-hand panel of Figure 2 shows the form of $F$ near to corotation $(m=2, k=0)$ at $t=200$, which is typical. Near to the resonance (marked by the vertical line), particles cross corotation in both directions on horse-shoe orbits (Binney \& Tremaine 1987, §7.5). The generally negative slope of $F$ implies an excess of lower $L$ particles that gain angular 


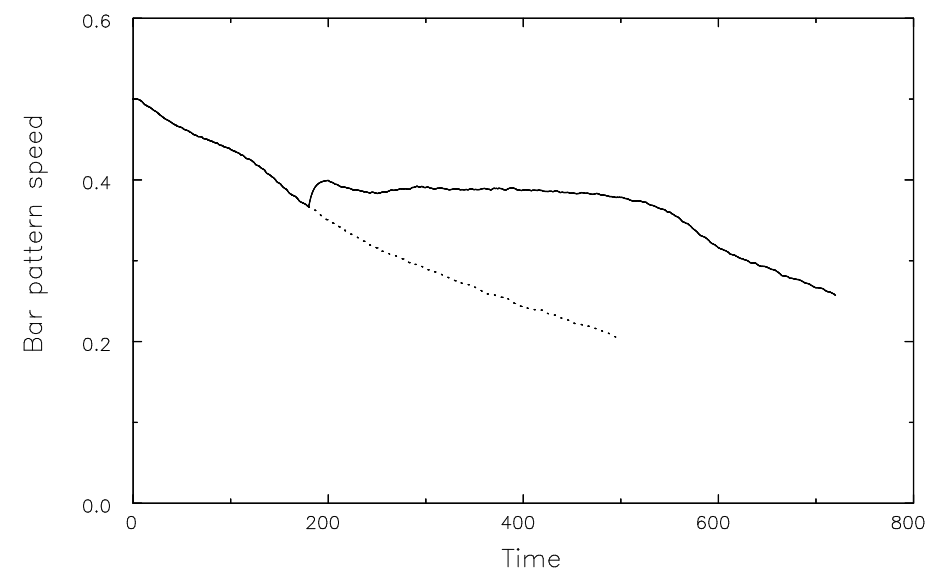

Figure 3. The solid curve shows evolution of the bar pattern speed in a restricted experiment in which the bar was reaccelerated (by external interference) between times 180 and 200, but was otherwise allowed to evolve freely. (This experiment used $1 \mathrm{M}$ particles.) The dotted curve shows what happens without interference.

momentum and move out across the resonance, and this imbalance is responsible for friction. If the pattern speed were to stay constant, the imbalance would tend to flatten the slope of $F$, and the distribution of particles about the resonance would approach kinetic equilibrium in which there would be more nearly equal numbers of particles crossing in both directions. But as $\Omega_{p}$ declines, the resonance keeps moving to larger $L_{\text {res }}$, and equilibrium is never established; instead, the density of particles about the dominant resonance(s) responsible for friction takes on the characteristic humped form shown in Fig. 2.

Friction arises principally at corotation over most of the evolution. The outer Lindblad resonance contributes in the early stages, but dominates only if the bar is unreasonably fast. The inner Lindblad resonance becomes important only when the bar is already quite slow; e.g. it is responsible for the more rapid braking around $t=1000$ in Fig. 2.

\section{Anomalous Situation}

Now suppose that $\Omega_{p}$ rises for some reason, after having declined for some time, as illustrated in Figure 3. The shoulder in $F$ created by the previous friction survives, but the resonance at the now higher $\Omega_{p}$ lies on the other side of the shoulder, as shown in Figure 4. Thus the local gradient in $F$ has changed sign, leading to an adverse gradient for friction, and the torque from the dominant resonance disappears. Under these circumstances, a balance between gainers and losers is soon 

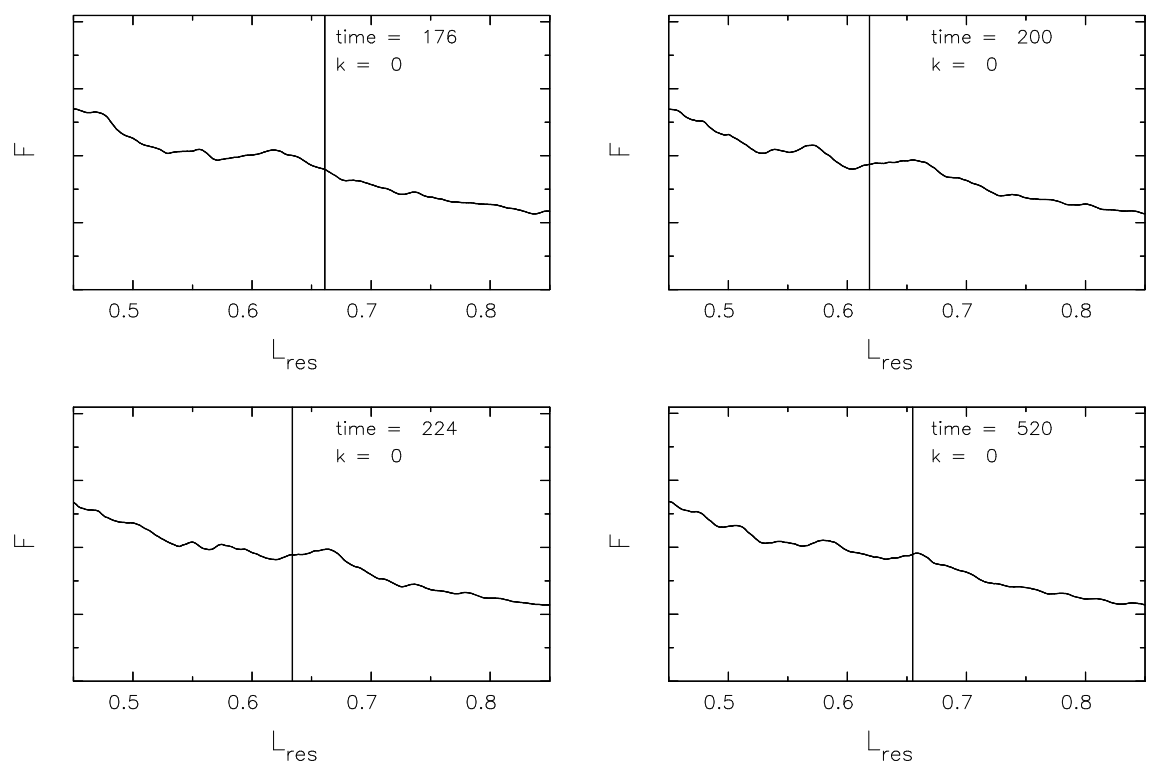

Figure 4. The mean density of particles as a function of $L_{\text {res }}$ at several different times in the simulation shown in Fig. 3.

established, and the bar can rotate in a dense halo with little friction, which we describe as a "metastable state".

In fact, $\Omega_{p}$ declines slowly because of weak friction at other resonances, and normal friction resumes when the slope of $F$ at the main resonance changes, as shown in the last frame of Fig. 4.

\section{Self-consistent Simulations}

If we now re-examine Fig. 1, we see that the period of weak friction is preceded by a small rise in the bar pattern speed in both the simulation of VK03 (dot-dashed line) and in the anomalous case we found. It is likely therefore that friction stopped for a while in both cases because the local density gradient across the principal resonance became flat, as just described.

Analysis of our simulation that displayed this behavior suggests that $\Omega_{p}$ rose because of an interaction between the bar and a spiral in the disk, which caused the bar to gain angular momentum. Such an event is rare; spirals generally remove angular momentum from the bar at most relative phases. It is possible that VK03 were unlucky to have such an event in their case, but they report similar behavior in their model B making a chance event unlikely. 
One significant difference between our code and that used by VK03 (Kravtsov, Klypin \& Khokhlov 1997) is that their resolution is adaptive, which causes gravity to strengthen at short range when the grid is refined. The increase in the local density as the bar amplitude rises causes the code to refine the grid, strengthening gravity and thereby causing the bar to contract slightly and to spin-up. We have found that a reduction of softening length in our code at this epoch also leads to a metastable state. It is likely, therefore, that their anomalous result is an artifact of their adaptive code.

\section{The Metastable State is Fragile}

Whatever the origin of the bar speed-up in simulations, it remains possible that the metastable state could occur in real galaxies. If friction in a dense halo can be avoided for this reason, then the observed pattern speeds will provide no constraint on the halo density.

However, further experiments in which we perturbed our model in the metastable state very slightly, revealed that the state is highly fragile. For example, a satellite of merely $1 \%$ of the galaxy mass flying by at $30 \mathrm{kpc}$ is sufficient to jolt the system out of the metastable state. We therefore conclude that anomalously weak friction is unlikely to persist for long in nature.

\section{Conclusions}

Tremaine \& Weinberg (1984) showed that angular momentum is transferred from a rotating bar to the halo through resonant interactions. We find that friction is dominated by a single resonance at most times, and that corotation is most important for a bar with realistic pattern speed - i.e. when the bar extends almost to corotation.

Friction arises because the phase space density is a decreasing function of angular momentum in normal circumstances, causing an excess of particles that gain angular momentum over those that lose. While this process would tend to flatten the density gradient if the pattern speed remained steady, the decreasing angular speed of the bar prevents this steady state from being reached. Instead we find that the density of particles in phase space develops a shoulder, with the resonance holding station on the high-angular momentum side of the shoulder as the feature moves to larger $L_{\text {res }}$.

However, if the bar is spun up slightly for some reason after a period of normal friction, the rise in the pattern speed may move the resonance to the other side of the pre-constructed shoulder. The change in the local gradient of particle density at the dominant resonance causes 
friction to become very weak for a while, allowing the bar to rotate almost steadily. Mild friction persists because of contributions from other, sub-dominant resonances, and normal friction resumes once the pattern speed has declined sufficiently for the gradient at the main resonance to become favorable for friction once more.

A state in which strong friction is suspended for this reason is "metastable", both because it relies on a local minimum in the phase space density, and because the state is fragile. A very mild jolt to the system is sufficient to cause normal friction to resume.

The absence of friction in the simulation $\mathrm{A}_{1}$ reported by Valenzuela \& Klypin (2003) is probably an artifact of their code. Their adaptive grid causes gravity to strengthen as the bar density builds up, making the pattern speed of the bar rise for a purely numerical reason. Thus their claimed counter-example to the argument of Debattista \& Sellwood $(1998,2000)$ is a numerical artifact of their method. Pace Holley-Bockelmann \& Weinberg (2005), our constraint on halo density still stands: A strong bar in a dense halo will quickly become unacceptably slow through dynamical friction.

\section{Acknowledgments}

We thank Anatoly Klypin for providing the initial positions and velocities of the particles in his model, and for many discussions. This work was supported by NASA (NAG 5-10110) and the NSF (AST-0098282).

\section{References}

Binney, J. \& Tremaine, S. 1987, Galactic Dynamics (Princeton: Princeton University Press)

Debattista, V. P. \& Sellwood, J. A. 1998, Ap. J. Lett., 493, L5

Debattista, V. P. \& Sellwood, J. A. 2000, Ap. J., 543, 704

Hernquist, L. 1990, Ap. J., 356, 359

Holley-Bockelmann, K., Weinberg, M. D. \& Katz, N. 2003, astro-ph/0306374

Holley-Bockelmann, K. \& Weinberg, M. D. 2005, DDA abstract 36.0512

Kalnajs, A. J. 1977, Ap. J., 212, 637

Kravtsov, A. V., Klypin, A. \& Khokhlov, A. M. 1997, Ap. J. Suppl., 111, 73

Lin, D. N. C. \& Tremaine, S. 1983, Ap. J., 264, 364

Lynden-Bell, D. 1979, MNRAS, 187, 101

Lynden-Bell, D. \& Kalnajs, A. J. 1972, MNRAS, 157, 1

Sellwood, J. A. 2003, Ap. J., 587, 638

Sellwood, J. A. 2004, astro-ph/0407533

Tremaine, S. \& Weinberg, M. D. 1984, MNRAS, 209, 729

Valenzuela, O. \& Klypin, A. 2003, MNRAS, 345, 406

Weinberg, M. D. 2004, astro-ph/0404169

Weinberg, M. D. \& Katz, N. 2002, Ap. J., 580, 627 